\title{
Diseño de un programa comunitario en una empresa de transportes de San Juan de Pasto 1
}

\author{
Maria Fernanda Burbano \\ Psicóloga \\ Universidad de Nariño, Colombia \\ Correo electrónico: mafesitab@gmail.com
}

\section{Katherine Stephany Córdoba Reina \\ Psicóloga \\ Universidad de Nariño, Colombia Correo electrónico: psicordobareina@gmail.com}

\author{
Álvaro Darío Dorado Martínez \\ Psicólogo \\ Universidad de Nariño, Colombia \\ Correo electrónico: \\ alvarodoradomartinez@gmail.com \\ Fredy Arley Ruano Bermúdez \\ Psicólogo \\ Universidad de Nariño, Colombia \\ Correo electrónico: freddyarleyr@gmail.com \\ Estefania Sabido Araujo \\ Psicóloga \\ Instituto Politécnico Nacional, México \\ Correo electrónico: fanysabido19@hotmail.com
}

Recibido: 26/04/2019

Evaluado: $10 / 07 / 2019$

Aceptado: 19/03/2020

\section{Resumen}

La interacción social no solo da cuenta de los comportamientos y actitudes que muestran los seres humanos para comunicarse, sino que además permite proponer marcos explicativos y de análisis sobre los procesos relacionales que caracterizan a las organizaciones. En este sentido, el artículo tiene como objetivo presentar el diseño de un programa de intervención comunitaria en una empresa de transportes de la Ciudad de San Juan de Pasto, Colombia. El estudio se realizó desde el paradigma cualitativo, con enfoque crítico social y tipo de estudio investigación acción. Para la recolección de información se utilizaron como técnicas la revisión de la literatura, la entrevista semiestructurada, grupo focal y observación participante. Los resultados evidencian la necesidad de implementar un programa enfocado en la autogestión, las habilidades sociales y el civismo en los colaboradores y los usuarios de la organización, lo cual contribuye no solo a su bienestar integral sino también al mejoramiento de los procesos organizacionales. Cabe aclarar que el presente artículo describe la fase de diseño de programa de intervención, la cual se seguirá desarrollando.

\section{Palabras clave}

Autogestión, civismo, interacción social, habilidades sociales, organización y usuario.

1 Para citar este artículo: Burbano, M., Córdoba, K., Dorado, A., Ruano, F. y Sabido, E. (2021). Diseño de un programa comunitario en una empresa de transportes de San Juan de Pasto. Informes Psicológicos, 21(1), 13-27 http://dx.doi.org/10.18566/infpsic.v21n1a01 


\section{Design of a community program in a transportation company in San Juan de Pasto}

Abstract

Social interaction not only accounts for the behaviors and attitudes shown by human beings in order to communicate, but it also allows proposing explanatory and analytical frameworks on the relational processes that characterize organizations. In this sense, the article aims to present the design of a community intervention program in a transportation company located in the City of San Juan de Pasto, Colombia. The study was carried out from the qualitative paradigm, with a social critical approach and an action research type of study. Literature review, semi-structured interview, focus group and participant observation were used as techniques to collect the information. The results show the need to implement a program focused on self-management, social skills and civility in employees and users of the organization, which contributes not only to their overall well-being, but also to the improvement of organizational processes. It should be noted that this article describes the intervention program design phase, which will continue to be developed.

\section{Keywords}

Self-management, civics, social interaction, social skills, organization and user.

\section{Desenho de um programa comunitário em uma empresa de transportes de San Juan de Pasto}

\section{Resumo}

A interação social não só responde pelos comportamentos e atitudes demonstrados pelo ser humano para se comunicar, mas também permite propor quadros explicativos e analíticos sobre os processos relacionais que caracterizam as organizações. Nesse sentido, 0 artigo tem como objetivo apresentar 0 desenho de um programa de intervenção comunitária em uma empresa de transporte na cidade de San Juan de Pasto, Colômbia. 0 estudo foi realizado a partir do paradigma qualitativo, com abordagem social crítica e estudo do tipo pesquisa-ação. Revisão da literatura, entrevista semiestruturada, grupo focal e observação participante foram utilizadas como técnicas de coleta de informações. Os resultados apontam para a necessidade de implantação de um programa voltado para a autogestão, as habilidades sociais e civilidade dos funcionários e usuários da organização, contribuindo não só para o seu bem-estar geral, mas também para a melhoria dos processos organizacionais. É de notar que este artigo descreve a fase de concepção do programa de intervenção, o qual continuará a ser desenvolvido.

Palavras chave Autogestão, civismo, interação social, habilidades sociais, organização e usuário. 


\section{ntroducción}

La psicología comunitaria analiza el modo en el que los individuos se relacionan con los grupos sociales de los que forman parte (Seidman, 2011), lo cual se ha ido transformando a través del tiempo con los procesos de urbanización, industrialización y el acceso masivo a internet dando pie a la aparición de nuevas formas de relaciones y de vida comunitaria. En esta lógica se parte del concepto de comunidad que, según Hillery (1955), contiene las siguientes características: (a) disponer de un espacio geográfico definido, (b) mantener vínculos comunes, y (c) interaccionar socialmente. Por su parte, Siles (2005) propone que la comunidad es un espacio social que va más allá de las condiciones geográficas y que permite a los individuos interactuar y encontrarse desde estas concepciones.

En lo concerniente al proceso de evaluación comunitaria en el contexto organizacional, éste es de fundamental importancia porque permite identificar, adquirir, analizar y dar informes sobre las fortalezas de la comunidad, sus recursos y necesidades para así buscar la manera de satisfacerlas. Asimismo, ayuda a encaminar los cambios y a motivar a la comunidad a actuar por sí misma (Rebollo, Morales \& González, 2016). Cabe resaltar que para el proceso óptimo de la evaluación comunitaria se deben tener en cuenta a todos los actores comunitarios que están determinados, a su vez, por las características sociodemográficas, biografía, historia de vida e interacción con el medio ambiente $y$, de esta manera, llegar a generar acciones que mejoren la calidad de vida y el bienestar desde una proyección multidisciplinar en la organización (Hernández \& Galindo, 2007).

En la presente investigación se parte de fenómenos propios de la comunidad, vinculados con un entorno en particular, que además involucra la colaboración de los participantes en la detección de necesidades, por cuanto conocen y han experimentado estos fenómenos. En general, el diseño de la investigación consta de tres fases cíclicas: (a) observar y recolectar datos sobre el problema, (b) analizar e interpretar, y (c) actuar para resolver el problema (Stringer, 1999, citado por Hernández, Fernández \& Baptista, 2010). Es así cómo, a través de las diferentes técnicas, se recolectó la información, se trianguló y se analizó. En este sentido, emergieron tres categorías de análisis deductivas, a saber: la autogestión, donde se encuentran las subcategorías comprensión crítica del contexto, participación y concertación; habilidades sociales, conformada por las subcategorías comunicación asertiva y habilidades sociales contextualizadas y, por último, la categoría civismo.

La autogestión se comprende como un proceso integral que a partir de diferentes herramientas permite a la comunidad desde su diversidad, entorno y el análisis crítico de los problemas, generar diferentes estrategias y recursos funcionales para la consecución de roles personales y colectivos. La diversidad de estos roles ofrece multidimensionalidad en la intervención y en los métodos, abordados desde la retroalimentación de la teoría existente y el saber experiencial (Brivio, 2001; Rojas, 2016).

Por su parte, las habilidades sociales se definen como el conjunto de conductas 
aprendidas, emitidas por un sujeto en una situación interpersonal, socialmente aceptadas y que posibilitan la interacción con los demás, permitiendo expresar sentimientos, actitudes, deseos, opiniones, derechos, entre otros (Vega, González, Anguiano, Nava, \& Soria, 2009). Además, dichas conductas deben estar en concordancia con el contexto social (Santos \& Lorenzo, 1999).

Los componentes de las habilidades son: comunicación asertiva y habilidades sociales contextualizadas; el primero hace referencia a la capacidad de expresarse verbal y preverbalmente en forma apropiada a la cultura y a las situaciones (Van-der Hofstadt Román, 2005); y el segundo, se refiere a habilidades sociales desde un marco cultural determinado, que depende y se adapta a los factores sociodemográficos, el estado de una persona y la situación particular en la que se encuentra (Meichenbaum, Butler \& Gruson, 1981, citados en Ortego, López, Alvarez, \& Aparicio, 2010).

En lo que confiere al civismo, éste hace alusión a una conducta que se desarrolla de acuerdo con las normas de convivencia que reglamentan la vida social de un sujeto. Se refiere a los principios que conforman la educación para la ciudadanía, es decir, una persona cívica es consciente de sus derechos y deberes, es libre, crítico, responsable, participativo y solidario. El civismo tiene como fin último inculcar en los seres humanos el respeto hacia lo individual, lo grupal y lo institucional desde la diferencia, para así disminuir el debilitamiento del vínculo social y la fragmentación de la sociedad (Koubi, 2004). Este posee dos componentes, por un lado, se encuentran las actitudes institucionales e intereses de la nación, y por otro lado los comportamientos específicos de los ciudadanos, lo que permite el ajuste y la adaptación frente a las normas de convivencia pública, es decir, que el sujeto que cumple con sus deberes como ciudadano posee mayor capacidad para respetar las leyes y contribuye al bienestar de la sociedad (Lizcano, 2012).

Estudios previos abordan las categorías mencionadas anteriormente. Con respecto a la autogestión se encuentran diferentes artículos desarrollados en el contexto organizacional como la investigación denominada: "Política y subjetividad: la tensión autogestión-delegación en empresas y fábricas recuperadas" (Fernández, López, Borakievich \& Ojám, 2015), la cual se desarrolló desde el paradigma cualitativo. En esta se señala cómo la construcción de espacios de acción colectiva, particularmente en sus modalidades más autogestivas y delegativas, hacen posibles nuevos modos de organización, participación y co-construcción. Esto posibilita la invención de novedosos modos de organización. Asimismo, el estudio elaborado por Heras-Monner-Sans (2015), demuestra cómo la autogestión es un proceso relacionado con el aprendizaje colectivo mediante el cual cada sujeto se articula con la propuesta colectiva, que debe responder al deseo de cada participante, como una práctica anclada al saber hacer y al deseo, en un trabajo conjunto, donde se dan interacciones complejas, que responden a la necesidad de crear algo nuevo y de esta forma a trasgredir el orden dado, y la posibilidad de significar distinto.

En relación con las habilidades sociales, se han desarrollado investigaciones como la de Veloso, Cuadra, Gil, Quiroz y Meza (2015), quienes lograron identificar 
cómo éstas influyen en el éxito laboral, debido a que facilitan la construcción de relaciones satisfactorias y eficientes entre los colaboradores, fortaleciendo el trabajo en equipo, la resolución de conflictos, el trabajo coordinado, cooperativo e integrado con los otros, contribuyendo así al incremento de la satisfacción laboral. Asimismo, el estudio realizado por Aquino et al. (2017) el cual tuvo como objetivo analizar las condiciones de trabajo en conductores de autobús: de servicio público a fuente de riesgo, en la ciudad de Recife (Brasil), logró evidenciar que una de las condiciones de trabajo que interfiere en la salud ocupacional de los conductores son las relaciones interpersonales con los pasajeros; específicamente un 48.3\% de las quejas se relacionan con experiencias o situaciones vivenciadas principalmente con los usuarios, de ahí la importancia de incluirlas y fortalecerlas en los procesos de intervención.

En lo que confiere al civismo, se han elaborado estudios como el de Arias, Garnica, Perdomo y Alarcón (2016) donde se buscaba diagnosticar factores de riesgo de los pasajeros de servicio público en la Ciudad de Neiva, en el cual se evidenció que un aspecto de mejora en la prestación del servicio fue la impaciencia y falta de respeto por parte de los conductores. De igual manera, en la investigación denominada "Valores organizacionales y civismo en las organizaciones" se encontró que los comportamientos cívicos en las organizaciones pueden predecirse desde los valores organizacionales, como la armonía, tolerancia, respeto y dominio (Porto \& Tamayo, 2005), temas relevantes para el diseño del programa de intervención comunitario.
De lo anterior se puede deducir que los contextos organizacionales en los cuales se evidencia la ausencia de autogestión, relaciones sociales en déficit y falta de civismo se han convertido en prácticas cada vez más naturalizadas que, aunque desatendidas por la sociedad en general, cobran mayor validez cuando influyen elementos como el estrés, la ansiedad, las cargas laborales y emocionales, como lo corroboran los estudios mencionados anteriormente. Teniendo en cuenta lo planteado, el objetivo del presente estudio de investigación es diseñar un programa de intervención comunitario. Para ello se plantearon los siguientes objetivos específicos: (a) caracterizar al usuario de la empresa de transportes de San Juan de Pasto; (b) realizar el concepto diagnóstico; y (c) diseñar la estructura del programa de intervención. Es importante mencionar que la empresa se caracteriza por prestar el servicio de transporte público terrestre intermunicipal de pasajeros, carga y encomiendas, así como también de distribuir a través de su estación de servicios, combustibles líquidos derivados del petróleo; además, tiene como visión ser una empresa líder, en continuo crecimiento, reconocida como modelo organizacional en el sur occidente colombiano. Se espera que mediante la caracterización, el diagnóstico y el diseño del programa de intervención referentes al fortalecimiento de la autogestión, las habilidades sociales y el civismo, la organización se convierta en un factor protector para esta comunidad y pueda generar estrategias que no solamente posibiliten la ampliación del portafolio de servicios, sino que desarrolle acciones de carácter comunitario (Restrepo de Ocampo, Estrada \& López, 2009). 


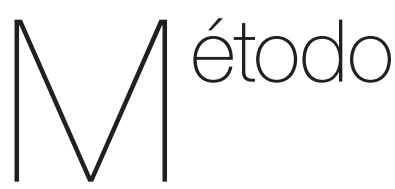

El presente estudio se desarrolló a través del paradigma cualitativo, el cual permitió vislumbrar la realidad desde la particularidad y facilitó la comprensión de los fenómenos propios de la comunidad como la interacción social (Corbin \& Strauss 2001; Taylor \& Bogdan 1987). Se abordó desde el enfoque crítico social, ya que permitió el desarrollo de la autonomía, encaminada a la toma de decisiones (Gómez \& Villalobos, 2014), y se orientó desde la investigación colaborativa, ya que este diseño se refiere a la colaboración entre investigadores responsables de programas específicos y miembros de una comunidad o grupo para el desarrollo de un estudio, con el fin de utilizar la investigación como un instrumento para resolver un problema y generar cambios sociales (López, 2010).

La unidad de análisis estuvo conformada por la comunidad de la empresa, que comprende usuarios y colaboradores de la misma; la unidad de trabajo para la caracterización estuvo conformada por 979 usuarios encuestados, y para el proceso de evaluación comunitaria participaron un total de 33 personas quienes fueron seleccionadas por un muestreo por conveniencia y de acuerdo a su disponibilidad. De la muestra, 11 sujetos pertenecían a las áreas de servicio al cliente y administrativos, 11 eran conductores y otras 11 personas eran usuarios del servicio de transporte. Se tomaron como criterios de inclusión el ser colaborador o usuario de la empresa y haber firmado el consentimiento informado, mientras que como criterios de exclusión se tuvo en cuenta a aquellos colaboradores que no tenían usual o permanente contacto y atención con el pasajero.

Como técnicas de recolección de información se utilizó, inicialmente, la revisión documental, con el fin de hacer lectura de la realidad y familiarizarse con la organización; para ello se revisaron 5 documentos correspondientes a la Tabulación Final Pasajeros, el Informe de PQRS 2017, Servicio al Cliente Informe año 2016, Medición de la Satisfacción del Cliente de Transporte de Pasajeros y Medición de la Satisfacción del Cliente.

Consecutivamente se realizó la entrevista semiestructurada a agentes claves (conductores, taquilleros, inspectores de patio), mediante la cual se aclararon términos, se identificaron ambigüedades y necesidades. Todos estos elementos fueron claves para el proceso de caracterización y para la aproximación al perfil del usuario, herramienta que servirá de apoyo en el diseño y desarrollo del programa comunitario.

Posteriormente, se realizó un grupo focal con conductores, dado que brinda un espacio de opinión para comprender el sentir, pensar y vivir de los colaboradores, desde las auto-explicaciones que permiten enriquecer la información (Hamui \& Varela, 2012). Complementario a ello se realizó la observación directa en la terminal de transporte, debido a la necesidad de recopilación de la información sin dirigirse a los sujetos involucrados, recurriendo desde el sentido observador del investigador que tiene como base una guía de observación que se crea a partir de elementos construidos previamente, que permiten vislumbrar los comportamientos 
que han de observarse (Martínez, 2007). Estos datos fueron recopilados en un diario de campo, permitiendo sistematizar la práctica de investigación a partir del fortalecimiento de la relación dialéctica (Martínez, 2007). Cabe resaltar que todas las técnicas mencionadas anteriormente se desarrollaron con un protocolo previo que cuenta con un saludo inicial, descripción del objetivo y la actividad, desarrollo de la técnica y cierre.

Finalmente, a los usuarios se les aplicó la técnica DOFA (Debilidades, Oportunidades, Fortalezas, Amenazas) para realizar una evaluación de los factores fuertes y débiles que en conjunto determinan la situación interna de una organización. Asimismo, se pueden identificar las oportunidades y amenazas con las que cuenta la empresa mediante una evaluación externa (Ponce-Talancón, 2006).

Para el análisis de los datos se realizó una triangulación de forma manual y se llevó a cabo el siguiente proceso: organización de la información recolectada, transcripción de resultados, construcción de las categorías de análisis, triangulación y análisis de los datos.

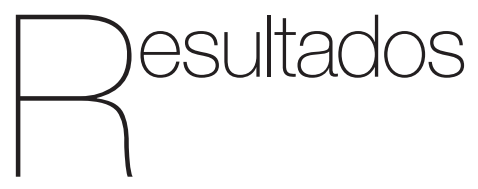

Los resultados encontrados permitieron reconocer que la organización busca desarrollar estrategias de mejoramiento continuo desde la participación y construcción de los diferentes actores; es por esta razón por la que cobra importancia el proceso de evaluación comunitaria en el contexto organizacional ya que posibilita la comprensión de las dinámicas entre colaboradores, el usuario y el servicio, desde una lectura de los fenómenos sociales dados en el contexto. A partir del análisis de las experiencias y sentires expresados y evidenciados, se logró inicialmente realizar la caracterización del usurario y consecutivamente la identificación de necesidades (concepto diagnóstico), lo que permitió diseñar una propuesta de intervención acorde a la realidad.

\section{Datos sociodemográficos del usuario}

Cabe mencionar que los datos sociodemográficos fueron obtenidos mediante el análisis del material proporcionado por la empresa tales como: Tabulación Final Pasajeros, Informe de PQRS 2017, Servicio al Cliente Informe año 2016, Medición de la Satisfacción del Cliente de Transporte de Pasajeros y Medición de la Satisfacción del Cliente. Es así como de las 979 encuestas diligenciadas, el 53.24\% pertenecen al género masculino y el $46.76 \%$ al género femenino, cuyas edades están comprendidas entre los 15 y 70 años, con una media de 34 años y una desviación estándar de 12.3043. Los estratos económicos a los que pertenecen son bajo y medio. Se encontró que frente a la ocupación en su mayoría son empleados, estudiantes y comerciantes.

En lo que refiere al uso del servicio, se identificó que la mayoría de los pasajeros proceden del Departamento de Nariño y su destino frecuente de viaje es la ciudad 
de Cali, Colombia. La línea de transporte de preferencia es la Línea Andina, con una frecuencia de uso que aumenta en temporadas especiales, como vacaciones, trabajo o familia. Es importante mencionar que hay usuarios con una frecuencia de uso diaria y semanal que han viajado con esta empresa por tres o más años.

\section{Caracterización del usuario}

A partir de la información brindada por los colaboradores de la empresa, se logró identificar que algunos usuarios son percibidos como "respetuosos, cordiales y amables" y otros como "poco asertivos, exigentes, impacientes y enojados"; también se reportó que "esto se podría determinar a partir de la presencia o ausencia de normas de cortesía, así como también, el cuidado y aseo interno del vehículo". De igual manera, se evidenció que la mayoría de los usuarios manifestaron observar diferentes tipos de actitudes entre las que se destacan actitudes "proactivas" y "preocupadas". Asimismo, "muchos de los sujetos tenían conductas que reflejaban ansiedad, especialmente cuando se trata de los equipajes, la falta de información sobre los horarios y la capacidad de carga".

Algunas percepciones de los colaboradores distinguieron al usuario entre dos tipos, categorizándolos a unos como "educados y amables", los cuales usualmente utilizan las líneas de mejor categoría como lo son Andina, Andina Duo y Andina Duo Plus; por otro lado, los usuarios que viajan en líneas económicas son percibidos como "poco asertivos comunicativamente y no mantienen el vehículo en óptimas condiciones de aseo"

\section{Evaluación comunitaria}

A través del tiempo esta empresa de transportes ha fortalecido sus procesos en cuanto a prestación de servicios y construcción de dinámicas de interacción social, las cuales le han permitido posicionarse como una organización que le apuesta al emprendimiento y la innovación social en sus acciones. Es por ello que este proceso de evaluación comunitaria ha llevado a entender la importancia del mejoramiento constante y la resolución de las necesidades encontradas, lo cual se realizará a través de un programa de intervención comunitaria que involucra el fortalecimiento de la autogestión, habilidades sociales y el civismo, apoyándose del uso estratégico del perfil de sus usuarios, pues estos son parte esencial de esta comunidad de transporte.

\section{DOFA}

Con el fin de complementar el diagnóstico, se desarrolló con los usuarios la herramienta de estudio DOFA, puesto que evalúa los factores internos y externos, que en conjunto determinan la situación interna de la comunidad, así como su evaluación externa, es decir, las oportunidades y amenazas.

En lo referente a las debilidades, en la mayoría de los usuarios se identificó una apreciación positiva de sí mismos y de los demás acerca del servicio que se brinda. Muchos reportaron que "el comportamiento del pasajero era adecuado y que pocas veces se presentaban dificultades con el servicio". Sin embargo, otros usuarios, que frecuentemente utilizan el servicio, identificaron dificultades en su 
experiencia, por ejemplo, "la falta de información del protocolo de viaje, la falta de aseo de muchos usuarios, las normas de cortesía, condiciones de algunos vehículos y mal estado de vías de acceso, pero sobre todo la ausencia del contacto usuario-conductor".

En cuanto a las oportunidades, los usuarios refieren que, para tener una mejor experiencia de viaje, es importante "el uso de material audiovisual o informativo dado que puede permitir tener claridades y recomendaciones a la hora de utilizar el servicio". También reconocen a "los vínculos y las relaciones sociales con otros usuarios como una forma de identificación y de diálogo, antes de abordar el autobús".

Las fortalezas se encuentran encaminadas hacia la efectividad y rapidez de los viajes, así como también a la "comodidad de los buses, permitiendo a los usuarios estar confortables".

Las amenazas más frecuentes se relacionan con "los accidentes de tránsito, debido al mal estado de las vías, las condiciones inseguras de algunos vehículos y el exceso de velocidad", sin embargo, la totalidad de ellos afirmaron no haber vivenciado un suceso de esta índole.

\section{Diseño del programa de intervención}

Mediante el proceso de reconocimiento y evaluación comunitaria se logró la identificación de ciertas necesidades, por lo cual se han definido las siguientes categorías: autogestión, habilidades sociales y civismo, las cuales se abordarán en la implementación del programa de intervención comunitario (ver Figura 1).

Dentro de la categoría Autogestión se encuentra la subcategoría comprensión crítica del contexto, en la cual se pudo identificar la necesidad de construir programas, estrategias interactivas o de marketing digital acordes a las características del usuario; por ello, se vislumbra la importancia de hacer un uso adecuado y estratégico desde la empresa de los datos sociodemográficos y la descripción del usuario, de esta manera será posible reconocer a la población a quienes estarán dirigidas las acciones. Por otro lado, está la subcategoría participación y concertación, en la cual se distinguió la inexistencia de paquetes turísticos que puedan ampliar el portafolio de servicios respecto a la línea andina, así como también ausencia de reforzamiento para clientes que viajan frecuentemente con la empresa, es por esto por lo que en el programa de intervención se propone desarrollar estrategias desde la interdisciplinariedad, involucrando diferentes actores y dependencias de la empresa (publicidad, tesorería, psicología, salud y seguridad en el trabajo), para que desde su cooperación logren reconocer los recursos que poseen y así ellos mismos puedan crear e implementar diferentes estrategias encaminadas a la mejora continua de este fenómeno.

\section{Habilidades Sociales}

La categoría Habilidades Sociales está conformada por dos subcategorías; la primera es la Comunicación asertiva en la cual se encontraron aspectos de mejora en componentes actitudinales a la hora de prestar el servicio, en espacios como la taquilla, el contacto en el momento de 
guardar el equipaje, servicio al cliente, entre otros contextos, evidenciando dificultades en las relaciones interpersonales entre colaboradores, y colaborador-usuario; además, se encontró poca efectividad en la comunicación de los protocolos de viaje, reflejando la necesidad de reforzar las habilidades comunicativas.

La segunda subcategoría hace alusión a las habilidades sociales contextualizadas, en las cuales se encontró que los colaboradores tienen un contacto frecuente con personas que asisten a procesos de salud, como tratamientos, citas médicas, cirugías, entre otras, para quienes les es difícil la movilidad y sufren dolores, sin embargo, no existen estrategias que permitan al colaborador prestar servicio a esta población.

Por otra parte, se encontró que dentro de sus labores, los conductores se han enfrentado a diferentes situaciones de peligro, como accidentes, robos, alteraciones de orden público, entre otras; es así como se identifica la necesidad de intervenciones que le posibiliten al colaborador estar capacitado en situaciones de crisis y logre reaccionar de forma adecuada ante ellas, generando bienestar en su persona y en las que lo rodean.
Las necesidades encontradas anteriormente, se abordarán en el programa de intervención con estrategias de educación enfocadas en temáticas como primeros auxilios psicológicos, resolución de conflictos, comunicación asertiva, tolerancia, entre otras, por medio de talleres de sensibilización, en constantes jornadas de capacitación dirigidas a colaboradores y campañas pedagógicas desde juego de roles dirigidas al resto de la comunidad.

\section{Civismo}

Con referencia al civismo, se identificó falta de cumplimiento de ciertas normas o valores, como falta de cortesía, irrespeto por parte de colaboradores pertenecientes a la organización hacia sus compañeros e incluso hacia los usuarios, y viceversa. Además, por parte de los usuarios también se logró vislumbrar el mal uso de las áreas comunes como los baños, sillones, pasillos, patios, así mismo, la falta de aseo en los espacios mencionados anteriormente. Frente a esta problemática se plantea desarrollar estrategias audiovisuales, juego de roles y jornadas de sensibilización que permitan generar una cultura de cuidado hacia los espacios de la comunidad.

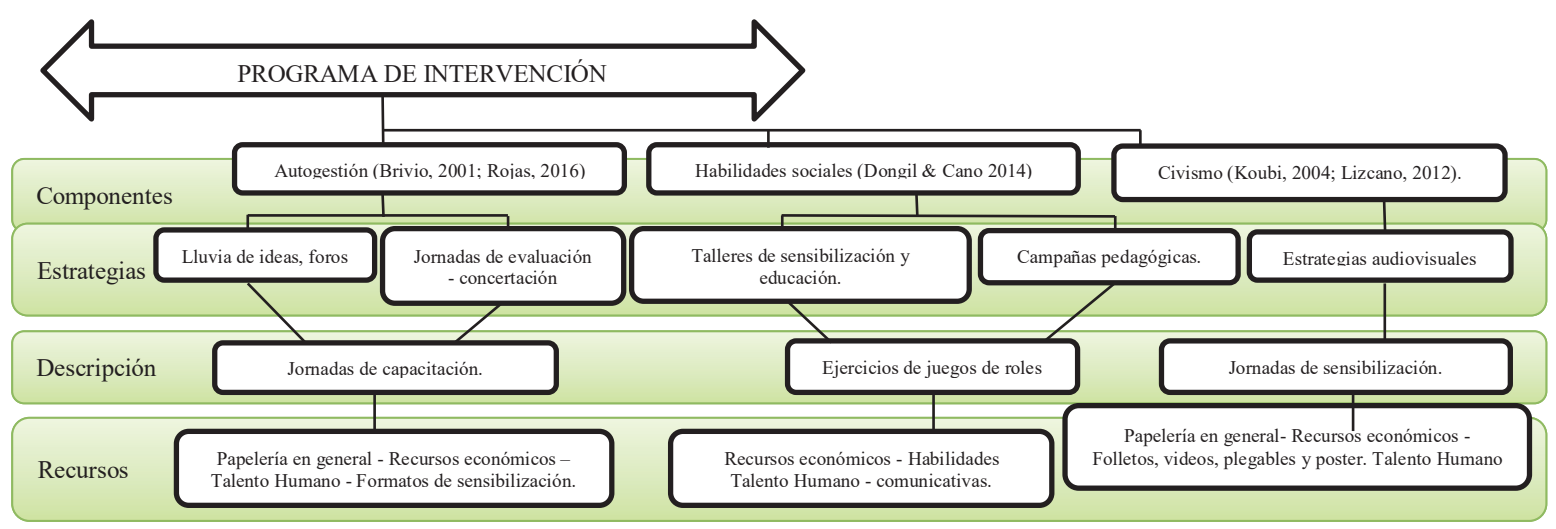

Figura 1. Estructura del programa de intervención en el usurario. 


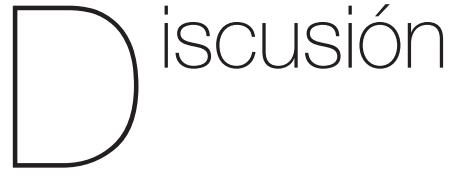

objetivo principal de la presente investigación fue diseñar un programa comunitario con el fin de identificar las necesidades de intervención en usuarios de una empresa de transportes de San Juan de Pasto, lo cual se logró a partir del análisis y triangulación de las diferentes técnicas utilizadas; primero se realizó la caracterización del usuario, fundamental para el desarrollo eficaz del programa de intervención, ya que como afirman Gallardo, Meneses y Minotta (2014) la caracterización de la población permite evidenciar las realidades sociales, políticas y culturales, logrando obtener conocimientos fiables del estado actual de las condiciones de vida de una sociedad, o una comunidad. Además, ofrece la posibilidad de comprender las dinámicas sociales de estas comunidades, donde no se limita solo a la recopilación de datos estadísticos, sino que también permite elaborar un diagnóstico de la realidad social de la comunidad con el fin de implementar programas específicos para determinadas poblaciones de una manera eficaz y contextualizada.

Por otra parte, los datos obtenidos en el proceso de caracterización se pueden contrastar con los resultados del estudio denominado "Diagnóstico de factores de riesgo de los pasajeros de servicio público en la Ciudad de Neiva" en el cual se encontró que el rango de edad más representativo está entre los 18-35 años, la frecuencia de uso de este servicio es de uso diario, la ocupación actual más representativa es ser trabajador independiente, trabajador vinculado y estudiantes con un nivel socioeconómico, uno y dos (Arias et al., 2016).

En cuanto al diseño inicial del programa comunitario, éste se encuentra enfocado en las categorías de autogestión, habilidades sociales y civismo. Con respecto a la autogestión, se logró identificar ausencia de programas y paquetes turísticos enfocados en las características del usuario. Frente a ello, Najul (2011) expone en su estudio que satisfacer las expectativas del cliente es muy importante y para ello es necesario disponer de toda la información precisa de los mismos para determinar el nivel de calidad de servicio y mantener a los colaboradores motivados con la intención de realizar carrera dentro de la organización, permitiendo una gestión de talento humano contextualizada e innovadora, acertada frente a los colaboradores y usuarios. Además se evidenció ausencia de reforzamiento para clientes frecuentes, mostrando la necesidad de trabajar conjuntamente con las diferentes dependencias y herramientas encontradas en la organización, con el propósito de generar bienestar para toda la comunidad y crecimiento organizacional (Brivio, 2001; Rojas, 2016).

Con respecto a la categoría de comunicación asertiva en las habilidades sociales se encontró un déficit en componentes actitudinales y comunicativos a la hora de prestar el servicio. Referente a esto, Ruiz-Arias, Nobles-Montoya y Ruiz-Otero (2015) encontraron que la asertividad indirecta, seguida de la no asertividad fueron los estilos de comunicación más utilizados por los funcionarios de atención al cliente. Estos factores propician que los individuos actúen en forma manipulativa, 
agresiva y/ o pasiva en su entorno social, resaltando la necesidad de trabajar en los estilos de respuesta utilizados en la comunicación con el fin de mejorar la calidad del servicio al cliente. Se plantea que la atención al cliente es trascendental para el éxito de cualquier organización, es un componente decisivo en la eficiencia de toda empresa y permite agregar valor a los servicios de la misma y esto se comprende desde Najul (2011) quien también define en su estudio la calidad del servicio al cliente, orientada desde la atención adecuada por parte de colaboradores altamente capacitados.

Por otro lado, con respecto a habilidades sociales contextualizadas, se logró evidenciar ausencia de programas que le permitan al conductor estar preparado en situación de crisis, eventualidades o necesidades especiales, ya sea en temáticas como primeros auxilios psicológicos, atención en crisis, entre otras. Así, un estudio realizado por Valencia y Trejos (2013) expone que los conocimientos sobre primeros auxilios psicológicos son una herramienta que le permite estabilizar emocionalmente a una persona que ha sido víctima de un evento inesperado (terremotos, accidente de tránsito, extorsión, hurto, secuestro, abuso de menores, intento de suicidio, sanciones de tránsito, entre otros), con el fin de evitar consecuencias negativas no solo para los implicados sino también para la comunidad observante; además, se vislumbra la necesidad de fortalecer la capacidad de interrelación de los colaboradores con sus pares, entre ellos principalmente conductores, servicio al cliente, taquilleros, y usuarios. Frente a lo último, Celis y García (2014) proponen que una gestión eficaz en el área de comunicación interna impacta positivamente en la organización, pues conforme van mejorando las relaciones de interacción interpersonal, el sentido de pertenencia y el vínculo con el público interno, es posible logar mayor productividad y, en esa medida, fortalecer la competitividad, rentabilidad y sostenibilidad de la organización.

Finalmente, se encuentra la categoría de civismo, referida al cumplimiento de ciertas normas o valores por parte de los colaboradores como de los usuarios en contexto (Lizcano, 2012), en este caso relacionado con el papel de la comunidad de transporte. Se logró identificar un déficit en la práctica de normas cívicas y valores en toda la presente comunidad, es decir, tanto de colaboradores como de usuarios. Frente a esto, Arias et al. (2016) identificaron en su estudio que un aspecto de mejora en la prestación del servicio, fue la impaciencia e irrespeto por parte de los conductores, así como otros estudios relacionados con los valores organizacionales como la armonía, tolerancia, respeto y dominio, implicando la reducción de conflictos y haciendo que el colaborador se sienta más seguro y dispuesto a cooperar con la organización (Porto \& Tamayo, 2005; Rocha-Dias \& De Fátima, 2016).

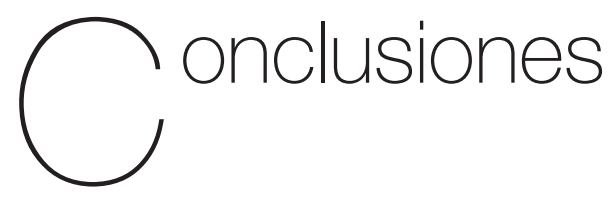

Se puede concluir que el proceso del diseño del programa de intervención social comunitario ha posibilitado el acercamiento a esta comunidad desde la integralidad; además, ha permitido generar 
espacios de reflexión entre la psicología comunitaria en un contexto organizacional, posibilitando la integración de herramientas, metodologías y saberes de evaluación para generar cambios profundos en las dinámicas organizacionales, así como también aportar evidencia novedosa acerca de un tema poco explorado en la literatura psicológica ya que no se encontró suficiente sustento teórico para contrastar la información recopilada.

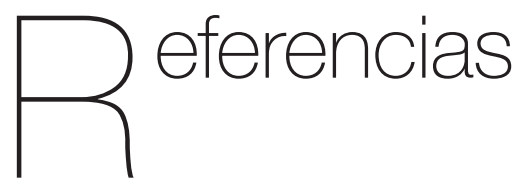

Aquino, J., Gomes de Medeiros, S., Mata, R., Betânia de Mata, R., Batista, E., Brandão, W. \& Gomes, M. (2017). Condiciones de trabajo en conductores de autobús: de servicio público una fuente de riesgo. Revista Index de Enfermería, 26(1-2), 34-38.

Arias, G., Garnica, P., Perdomo, Y. \& Alarcón, A. (2016). Diagnóstico de factores de riesgo de los pasajeros de servicio público en la Ciudad de Neiva. Revista UTP. Recuperado de http://revistas.utp.ac.pa/index.php/ memoutp/article/view/1268/html

Brivio A. (2001). La Autogestión comunitaria. Gestiópolis. Bogotá, Colombia. Recuperado de https://www.gestiopolis. com/la-autogestion-comunitaria/

Celis, E. \& García, C. (2014). La incidencia de la gestión de la comunicación en la estrategia corporativa. Informes Psicológicos, 14(1), 59-64. Recuperado de https://revistas.upb. edu.co/index.php/informespsicologicos/ article/view/3073/2706
Corbin, J. \& Strauss, A. (2001). Bases de la investigación cualitativa. Técnicas y procedimientos para desarrollar la teoría fundamentada. Editorial Universidad de Antioquia.

Fernández, A., López, M., Borakievich, S. \& Ojám, E. (2015). Política y subjetividad: la tensión autogestión-delegación en empresas y fábricas recuperadas. Facultad de Psicología - UBA. Secretaría de investigaciones. Anuario de investigaciones. Volumen XV.

Gallardo, N., Meneses, Y. \& Minotta, C. (2014). Caracterización poblacional vista desde la perspectiva del desarrollo humano y enfoque diferencial. Revista Investigación y Desarrollo, 22(2), 360-401.

Gómez, Y. \& Villalobos, F. (2014). Competencias para la formulación de un proyecto de investigación, guía metodológica para docentes investigadores. INVESTIC. San Juan de Pasto: Editorial Universidad de Nariño.

Hamui, A. \& Varela, M. (2012). La técnica de grupos focales. Departamento de Investigación Educativa, División de Estudios de Posgrado, Facultad de Medicina, Universidad Nacional Autónoma de México, México D.F., México.

Heras-Monner-Sans, A. (2015). Análisis del aprendizaje sobre la autogestión: La cuestión específica de los aportes y distribución de recursos. Cuadernos de Antropología Social, (41), 129-148.

Hernández, R., Fernández, C. \& Baptista, P. (2010). Metodología de la Investigación (5a. Ed.). México: Mc Graw Hill. 
Hernández, Y., \& Galindo, R. (2007). El concepto de intersubjetividad en Alfred Schutz. Espacios Públicos, 10(20), 228-240.

Hillery, G. A. (1955). Definitions of Community: Areas of Agreement. Rural Sociology, 20(1), $111-123$

Koubi, G. (2004). Entre "civismo" y "civilidad". La educación de la Ciudadanía. Revista Anales de la cátedra Francisco Suaréz, 38, 47-70.

Lizcano, F. (2012). Conceptos de ciudadano, ciudadanía y civismo. Polis (Santiago), 11(32), 269-304.

López, H. (2001). Investigación cualitativa y participativa. Un enfoque históricohermenéutico y crítico-social en psicología y educación ambiental. Facultad de Psicología, Universidad Pontificia Bolivariana.

Martínez, A. (2007). La observación y el diario de campo en la definición de un tema de Investigación. Perfiles libertadores. Recuperado de https:// escuelanormalsuperiorsanroque.files. wordpress.com/2015/01/9-la-observaciny-el-diario-de-campo-en-la-definicin-deun-tema-de-investigacin.pdf

Najul, J. (2011). El capital humano en la atención al cliente y la calidad de servicio. Observatorio Laboral Revista Venezolana, 4(8), 23-35.

Ortego, M., López, S., Álvarez, M., \& Aparicio, M. (2010). Ciencias Psicosociales I. Universidad de Cantabria. Recuperado de https://ocw.unican.es/course/view. php?id=173\&section $=1$
Ponce-Talancón, H. (2006). "La matriz FODA: una alternativa para realizar diagnósticos y determinar estrategias de intervención en las organizaciones productivas y sociales" en Contribuciones a la Economía.

Porto, J. \& Tamayo, Á. (2005). Valores organizacionais e civismo nas organizações. Revista de Administração Contemporânea, 9(1), 35-52.

Rebollo, O., Morales, E. \& González, S. (2016). Guía operativa de la evaluación de la acción comunitaria. Universidad Autónoma de Barcelona.

Restrepo de Ocampo, L., Estrada, S. \& López, M., (2009). Los seres humanos en la transformación organizacional. Revista Scientia Et Technica, 42(2), 351-356.

Rocha-Dias, F. \& de Fátima-Oliveira, Á. (2016). Valores e Confiança Organizacionais: Fatores Determinantes nos Comportamentos de Civismo Organizacional. Temas em Psicologia, 24(3), 1087-1100.

Rojas, C. (2016). De la autogestión comunitaria a lo sentipensante. Revista de Sociología y Antropología: VIRAJES, 18(2), 65-74.

Ruiz-Arias, V., Nobles-Montoya, D. \& RuizOtero, C. (2015). Asertividad en funcionarios de la administración pública del municipio de Montería, Colombia. Encuentros, 13(2), 13-24.

Santos, A. \& Lorenzo, M. (1999). La vía comunitaria en las instituciones cerradas: Un programa educativo de habilidades sociales. Revista de Ciencias de la Educación, 177, 79-96. 
Seidman, E. (2011). An Emerging Action Science of Social Settings. American Journal of Community Psychology, 50(1-2), 1-16.

Siles, I. (2005). "Internet, virtualidad y comunidad". Revista Ciencias Sociales, 2(108), 55-69. Recuperado el 15 de Junio de 2019 en http://www.redalyc.org/articulo. oa?id=15310805

Taylor, S. \& Bogdan, R. (1987). Introducción a los métodos cualitativos. Buenos Aires, Argentina: Ediciones Paidós.

Valencia, A. \& Trejos, J. (2013). Los primeros auxilios psicológicos en el servicio de atención al ciudadano desde un enfoque humanista. Revista Logos, Ciencia \& Tecnología, 4(2), 42-52.
Van-der Hofstadt Román, C. J. (2005). El libro de las habilidades de comunicación. Madrid: Díaz de Santos.

Vega, V., González, F., Anguiano, S., Nava, C. \& Soria, R. (2009). Habilidades sociales y estrés infantil. Revista Journal of Behavior, Health \& Social Issues, 1(1), 7-13.

Veloso, C., Cuadra, A., Gil, F., Quiroz, Á. \& Meza, S. (2015). Capacitación en trabajadores: impacto de un programa, basado en psicología positiva y habilidades sociales, en satisfacción vital, satisfacción laboral y clima organizacional. Interciencia: Revista de Ciencia y Tecnología de América, 40(11), 736-743. 